\title{
EVALUATION OF LDH AND GAMMA INTERFERON AS BIOCHEMICAL MARKERS FOR DIAGNOSIS OF PULMONARY AND EXTRA-PULMONARY TUBERCULOSIS
}

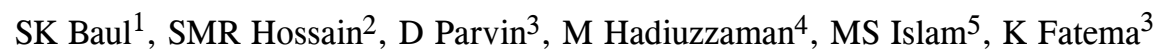 \\ ${ }^{1}$ Dept of Biochemistry, Satkhira Medical College, Satkhira; ${ }^{2}$ Dept of Microbiology, Sheikh Hasina Medical \\ College, Tangail; ${ }^{3}$ Dept of Clinical Biochemistry, NICVD, Dhaka; ${ }^{4}$ Dept of Biochemistry, Sheikh Shayera \\ Khatun Medical College, Gopalgon; ${ }^{5}$ Planning and Research, DGHS, Mohakhali, Dhaka
}

\begin{abstract}
Tuberculosis is a common disease throughout the world, especially in developing countries. The most common form of the disease is pulmonary tuberculosis (PTB). Extra pulmonary tuberculosis (EPTB) is also a growing problem worldwide. The diagnosis of PTB and EPTB is even harder when smears and cultures are negative. Due to its nature, the diversity of clinical pictures, the diagnosis of EPTB is often difficult and late. Advances in rapid diagnostic techniques are urgently required both for the early management of the new cases of TB and for the individuals already infected with Mycobacterium tuberculosis and are at risk of developing disease. In addition to standard TB diagnostic techniques, use of new biochemical surrogate markers like LDH and gamma interferon are new suggested. This study was undertaken to evaluate the diagnostic value and accuracy of serum LDH and gamma interferon for the diagnosis of PTB and EPTB. This crosssectional study was carried out in the Department of Pathology and Microbiology, NIDCH, Dhaka and by convenient and purposive sampling, 131 patients (male 98, female 33) having age range of 20-90 years were enrolled for the study. Serum LDH and QuantiFERON-TB gold (QFT-G i.e. gamma interferon) were estimated and the result was compared with the culture and histopathology results. Performance tests were done. Prevalence was measured at 95\% CI. Statistical significance was set at $\mathrm{p}<0.05$. Of 131 patients $89(67.9 \%)$ had pulmonary tuberculosis (PTB) and the rest 42 (32.1\%) had extra-pulmonary tuberculosis (EPTB). Level of LDH and gamma interferon in serum of all patients was found significantly high. TB pleural effusion was detected in $64 \%$ cases. The sensitivity, specificity, positive predictive values, negative predictive values and accuracy of LDH and gamma interferon wre calculated both in PTB and EPTB. For LDH it was (SEN 89.22\%, SPE $85.66 \%$, PPV 88.82\%, NPV 86.11\%) and for gamma interferon (SEN 93.01\%, SPE 91.64\%, PPV 93.73\%, NPV 91.18\%) in PTB; and for LDH (SEN 89.27\%, SPE 87.18\%, PPV 84.35\%, NPV 90.36\%) and for gamma interferon (SEN 94.88\%, SPE 93.65\%, PPV 90.04\%, NPV $95.82 \%$ ) in EPTB respectively. When calculated in combination, the sensitivity and specificity was $100 \%$ and $50 \%$ respectively in both PTB and EPTB, and positive and negative predictive values of LDH and gamma interferon in combination were $94.00 \%$ and $91.58 \%$ in PTB and $90.43 \%$ and $96.71 \%$ in EPTB respectively. The study concludes that the diagnostic accuracy of LDH and gamma interferon is low when single test is done but it is significantly high when done in combination.
\end{abstract}

Key Words: PTB, EPTB, LDH, QFT-G 


\section{Introduction}

Tuberculosis is a common disease throughout the world, especially in developing countries. Millions of people die each year from tuberculosis (TB), a leading chronic infectious killer of all age groups and the second most common infectious disease worldwide. It is an infection with human strains of Mycobacterium tuberculosis ${ }^{1,2}$. The most common form of the disease, which is responsible for the spread of $\mathrm{TB}$, is pulmonary tuberculosis (PTB). Tuberculosis can affect any organ in the body. Extra-pulmonary tuberculosis (EPTB) is referred to as isolated TB at any site in the body outside the lungs. Although TB can be fully cured with the use of appropriate drugs, the major hurdle to treatment for TB lies in the late diagnosis of the disease due to the lack of simple and cost-effective diagnostic procedures. Although the isolation of tuberculous bacilli from clinical specimens is the gold standard for diagnosis, chest $\mathrm{X}$ ray and the histopathological detection of granulomatous lesions in addition to clinical findings generally lead to true diagnosis. The diagnosis of PTB and EPTB is even harder when smears and cultures are negative. Extra pulmonary tuberculosis (EPTB) is a growing problem worldwide ${ }^{1-3}$ and due to the nature of the disease, diversity of clinical pictures as well as its minor epidemiological importance, the diagnosis is often difficult and late. Definitive diagnosis of tuberculosis includes a demonstration of the presence of $\mathrm{M}$. tuberculosis by microbiological culture and histopathological methods. Classical methods of TB diagnostics have significant limitations for diagnosis of EPTB. Culture is a lengthy process, while these patients often requires rapid diagnosis. Material for PCR diagnosis is often not possible to obtain (except cerebrospinal fluid and urine), and histopathological diagnosis requires surgical procedure, which is an invasive technique. The most reliable diagnostic criteria, is still to confirm the presence of bacilli in the patient's material by culture. In some forms of EPTB, material can be taken for culture from renal, meningeal, pleural, and pericardial fluid. Pleural effusion is a common reason for admission in hospitals and tuberculosis is found most common infectious cause. Pleural tissue histopathology and culture for Mycobacterium tuberculosis is considered the gold standard but the procedure is invasive, requires skilled clinicians and does not provide immediate results $2,4-9$. Advances in rapid diagnostic techniques are urgently required both for the early management of the new cases of TB and for the individuals already infected with Mycobacterium tuberculosis who are at risk of developing disease. In addition to standard TB diagnostic techniques, use of new biochemical surrogate markers (ADA, LDH, gamma interferon) are increased. An immunological test depending on the measurement of levels of gamma-interferon, the QuantiFERON-TB gold (QFT-G), specific to M. tuberculosis antigen produced by $\mathrm{T}$ cells have been developed in recent years. It is somewhat faster and more reliable test for diagnosing latent and active tuberculosis. Since there is no single test to diagnose $M$. tuberculosis infection, invasive procedures are needed in such cases, and diagnosis is usually delayed $8,10-23$. Therefore, to address these limitations, we evaluated lactate dehydrogenase (LDH) and gamma interferon in serum and to find out the combined efficacy of these two biochemical markers for rapid diagnosis of PTB and EPTB.

\section{Materials and Methods}

This hospital based cross-sectional study was conducted in National Institute of Diseases of the Chest and Hospital (NIDCH), Mohakhali, Dhaka at Pathology and Microbiology Department during the period of July 2014 to December 2014. After getting the approval of the research proposal from the ethical committee of the Institution and BMRC, consent was taken from each individual prior to inclusion. From selected patients, after aseptic precaution $5 \mathrm{ml}$ of whole venous blood from antecubital vein was collected in clean and dry test tube and was kept standing for clotting. Then it 
Evaluation of LDH and Gamma Interferon as Biochemical was centrifuged at $3000 \mathrm{rpm}$ to separate serum. Three samples of adequate amount of sputum were collected in a dry clean plastic cup from all the patients. The subjects were patients from OPD and indoor of Institute of Diseases of the Chest and Hospital (NIDCH), who were clinically suspected TB patients, hospitalized for investigation for fever of unknown origin. In addition to standard examinations, X- ray of the lungs, USG of abdomen, hematological and biochemical tests, abdomen, chest or pelvic CT scan, echocardiography was done depending on the presentation of illness. The histological processing of tissues of clinical interest (biopsy of the liver, lymph nodes, peritoneum, small bowel and adnexa) were also done. Histological processing of tissue samples was performed at the Pathology department of the institute. LDH and Gamma interferon (using QFT-G) in serum of all patients were measured by using standard procedure and method. After collection, all the data was checked and edited. Then data was entered into computer with the help of software SPSS for windows version 12. After frequency run, data was cleaned and frequencies were checked. An analysis plan was developed keeping in view with the objectives of the study (CI 95\%, p<0.05). Due to the heterogeneity of data, although the observed parameters were continuous, we used nonparametric tests ( $\chi^{2}$ test). Sensitivity and specificity of test results were calculated.

\section{Results}

For this study 208 clinically suspected tuberculosis (pulmonary and extra-pulmonary) patients were enrolled. Among them 131 patients (male 98, female 33) were proved to have tuberculosis by any one or in combination of sputum smear (Z-N or Auramine stain), sputum or tissue culture and histopathological examination. Age of the study subjects was between 20 to 90 years. Mean $( \pm S D)$ age of study subjects was $44.65 \pm 17.84$ years, that in male and female were $44.66 \pm 17.94$ and $44.61 \pm 17.77$ years.
Distribution of male and female is shown in Figure-1.

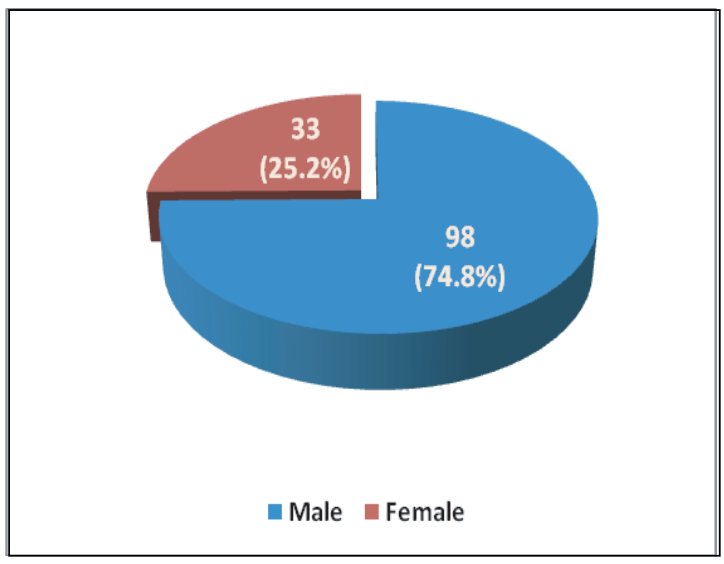

Figure-1: Sex distribution of the study subjects.

Table-I: Demographic Profile of the study Subjects

\begin{tabular}{lccccc}
\hline & & Male & \multicolumn{3}{c}{ Female } \\
\cline { 3 - 6 } *Income & & $\mathrm{n}=98$ & $(74.8 \%)$ & $\mathrm{n}=33(25.2 \%)$ \\
& Low & 59 & $(74.8 \%)$ & 23 & $69.7 \%$ \\
& Medium & 31 & $60.2 \%$ & 10 & $30.3 \%$ \\
Education & High & 08 & $31.6 \%$ & 00 & $00.0 \%$ \\
& Primary & 68 & $08.2 \%$ & 24 & $72.7 \%$ \\
& SSC & 22 & $69.4 \%$ & 07 & $21.2 \%$ \\
Smoking Habit & Smoker & 87 & $03.1 \%$ & 04 & $12.1 \%$ \\
& HSC & 05 & $22.4 \%$ & 02 & $06.1 \%$ \\
Residence & Non-smoker & 11 & $88.8 \%$ & 29 & $87.9 \%$ \\
& Kancha & 57 & $58.2 \%$ & 24 & $72.7 \%$ \\
& Semipacca & 38 & $38.7 \%$ & 09 & $27.3 \%$ \\
& Pacca & 02 & $03.1 \%$ & 00 & $00.0 \%$ \\
\hline
\end{tabular}

*Income (BD Tk./month): Low <10,000/-; Medium > 10,000/- to <30,000/-; High >30,000/-.

Demographic Profile of the study subjects are shown in Table-I. High frequency was found in low income, low education, smokers and dwellers of kancha residence among both male and female. 
72 Bangladesh J Med Biochem 2017; 10(2)

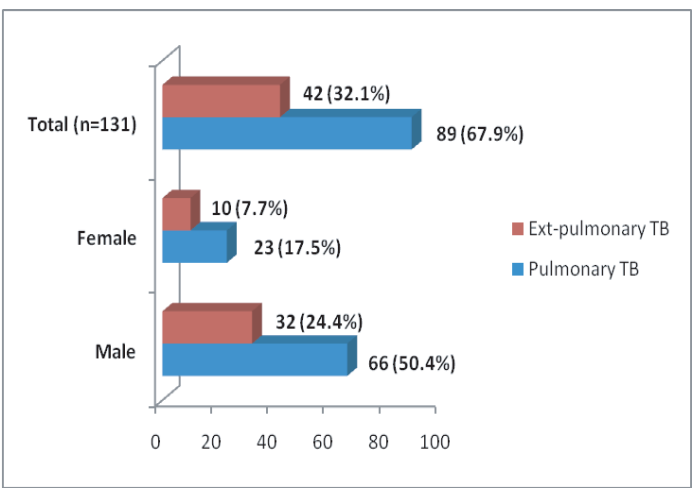

Figure-2: Disease distribution of the study subjects.

Eighty-nine $(67.9 \%)$ of total 131 patients had pulmonary TB and of them $66(50.4 \%)$ was male, $23(17.5 \%)$ was female. Other patients had extra-pulmonary TB. Among the pulmonary TB positive patients $74.2 \%$ was male and $25.8 \%$ was female whereas that of extra-pulmonary TB it was $76.2 \%$ and $23.8 \%$ respectively. No significant difference between types of diseases found between male and female (Figure-2 and 3).
SK Baul, SMR Hossain, D Parvin et al

Table-II: Results of Biochemical markers in Pulmonary TB \& Extra-Pulmonary TB

\begin{tabular}{lccc}
\hline & $\begin{array}{c}\text { Pulmonary TB } \\
(\mathbf{n}=\mathbf{8 9}) \text { number (\%) }\end{array}$ & $\begin{array}{c}\text { Extra-Pulmonary } \\
\text { TB(n=42) } \\
\text { number (\%) }\end{array}$ & $\begin{array}{c}\text { Total(n=131) } \\
\text { number (\%) }\end{array}$ \\
\hline S. QFT-G & & & \\
Positive & $73(82.0)$ & $33(78.6)$ & $106(80.9)$ \\
Negative & $16(18.0)$ & $09(21.4)$ & $25(19.1)$ \\
S. LDH & & & \\
Positive & $66(74.2)$ & $30(71.4)$ & $96(73.3)$ \\
Negative & $23(25.8)$ & $12(28.6)$ & $35(26.7)$ \\
Both S. QFT-G & & & \\
\& S. LDH & & & \\
Positive & $88(98.9)$ & $40(95.2)$ & $128(97.7)$ \\
Negative & $01(01.1)$ & $02(04.8)$ & $03(02.3)$ \\
& & & \\
\hline
\end{tabular}

Table-II shows the results of individual serum Quanti FeronTB-Gold (QFT-G), serum lactate dehydrogenase (LDH) and combined result of all the two biomarkers. When combined, the biomarkers show a high number of positive results, 128(97.7\%) out of 131 .

Table-III: SEN, SPE, PPV, NPV \& Accuracy of the Biochemical markers

\begin{tabular}{lcccccccccc}
\hline \multirow{2}{*}{$\begin{array}{l}\text { Biochemical } \\
\text { markers }\end{array}$} & \multicolumn{4}{c}{ Pulmonary TB } & \multicolumn{5}{c}{ Extra-Pulmonary TB } \\
\cline { 2 - 11 } & SEN & SPE & PPV & NPV & Acc & SEN & SPE & PPV & NPV & Acc \\
\hline S. LDH & 89.2 & 85.7 & 88.8 & 86.1 & 78.7 & 89.3 & 87.2 & 84.4 & 90.4 & 73.8 \\
S. $\boldsymbol{\gamma}$-Interferon (QFT-G) & 93.0 & 91.6 & 93.7 & 91.2 & 74.2 & 94.9 & 93.7 & 90.0 & 95.8 & 71.4 \\
Both S. QFT-G \& S. LDH & 100.0 & 50.0 & 98.9 & 100.0 & 98.9 & 100.0 & 50.0 & 95.0 & 100.0 & 97.7 \\
\hline
\end{tabular}

All results are in \%. SEN (Sensitivity), SPE (Specificity), PPV (Positive predictive value), NPV (Negative predictive value), Acc (Accuracy)

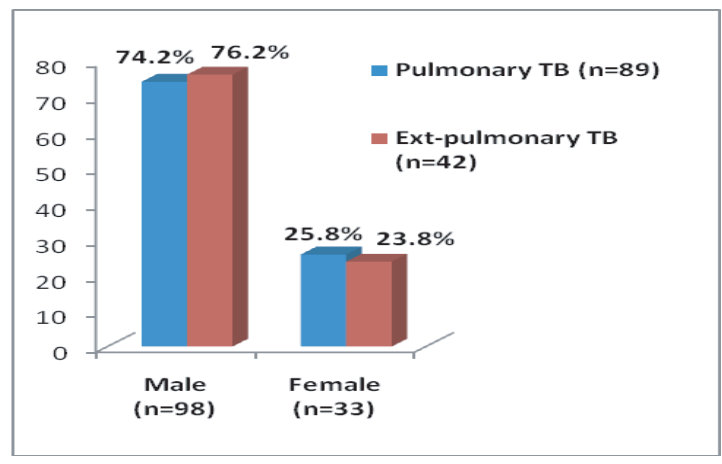

Figure-3: Distribution of Study Subjects according to disease conditions
Sensitivity, specificity, positive predictive value, negative predictive value and accuracy of serum Quanti FeronTB-Gold (QFT-G), serum lactate dehydrogenase (LDH) and those of combined of all the two biomarkers have been shown in Table-III. Each biomarker individually and all in combination showed good sensitivity and specificity.

\section{Discussion}

This hospital based cross-sectional study was conducted enrolling 208 clinically suspected tuberculosis (pulmonary and extra-pulmonary) 
Evaluation of LDH and Gamma Interferon as Biochemical

patients of 20-90 years of age, among them 131 patients were proved to have tuberculosis by any one or in combination of sputum smear (Z-N or Auramine stain), sputum or tissue culture and histopathological examination. In our study, like other several previous studies, demographic profile of the diseased persons and the prevalence of the disease in male and female were consistent ${ }^{1-9}$. Definitive diagnosis of tuberculosis includes a demonstration of the presence of M. tuberculosis by microbiological, cytological or histopathological methods. But, classical methods of TB diagnostics have significant limitations for diagnosis of EPTB. Cultivation is to long, while these patients often requires rapid diagnosis. Material for PCR diagnosis is often not possible to collect and histopathological confirmation requires biopsies, which are invasive. The most reliable diagnostic criteria, is still to confirm the presence of bacilli in the patient material by cultivation $9,10-18$. In some forms of EPTB material can be taken for culture but, unfortunately, successful culture is very variable19-23. Because of these problems in exact diagnosis of tuberculosis, numerous additional tests are in use with the intention to facilitate the diagnosis. In the recent years, biochemical markers for tuberculosis infection, such as levels of serum LDH or the level of interferon gamma is found to be much diagnostic importance. Serum LDH still remains as one of the important parameters to assess the cell/tissue specific stress or pathology, caused by Mycobacterium tuberculosis 24 .

In our study, we also found higher concentration of LDH in the serum of both PTB and EPTB patients. Our finding is consistent with that of Sharma et al. ${ }^{24}$. Some studies were done to assess the usefulness of gamma interferon estimation for diagnosis and management of immunocompromised individuals, in children and in those with extrapulmonary TB25. Our study also demonstrated the strong evidence of
QFT-G to diagnose both PTB and EPTB. Performance test of each biomarker was done to see the SEN, SPE, PPV and NPV to evaluate the efficacy of the biomarkers.

In conclusion, the diagnostic accuracy of LDH and gamma interferon may be low when single test is done. But, when they are used in combination, it is significantly high. Biochemical tests mentioned above may be useful adjunct tests for diagnosing TB and EPTB for early and appropriate management to reduce morbidity and mortality. Larger studies are needed in our country to determine more accurately the diagnostic value of new biomarkers, for PTB and EPTB.

\section{References}

1. World Health Organization. Global tuberculosis control: surveillance, planning, financing. Geneva: WHO, 2008.

2. Nelson LJ and Wells CD. Global epidemiology of childhood tuberculosis. Int J Tuberc Lung Dis 2004; 8(5): 636-47.

3. Raviglione MC, Snider DE Jr. Kochi A. Global epidemiology of tuberculosis: Morbidity and mortality of a worldwide epidemic. JAMA 1995; 273(3): 220-6.

4. World Health Organization. Global tuberculosis control: epidemiology, strategy, financing: WHO report 2009. Geneva: WHO, 2009.

5. World Health Organization. Tuberculosis fact sheets - TB and children. WHO, 2006.

6. National Tuberculosis Control Program. National guidelines and operational manual for tuberculosis control. $4^{\text {th }}$ Edition. Dhaka: Directorate General of Health Services, Ministry of Health \& Family Welfare, Bangladesh, 2009.

7. Zaman K, Yunus M, Arifeen SE, Baqui AH, Sack DA. Prevalence of sputum smear positive tuberculosis in a rural area in Bangladesh. Epidemiol Infect 2006; 134(5): 1052-9. 
74 Bangladesh J Med Biochem 2017; 10(2)

8. Narain JP, Pontali E, Tripathy S. Symposium on HIV and TB epidemiology and control strategies. Ind J Tuberc 2002; 49: 3-8.

9. Global Tuberculosis Control, WHO report, 2009.

10. Fanning A. Tuberculosis: Extrapulmonary disease. CMAJ 1999; 160: 1597-603.

11. Iscman MD. Tuberculosis in relation to human immunodeficiency virus and acquired immunodeficiency syndrome. In: Iseman MD, editor. A clinician's guide to tuberculosis. Philadelphia: Lippincott Williams and Wilkins; 2000 p. 199-252.

12. Lein $A D$, Von Reyn CF. In vitro cellular and cytokine responses to mycobacterial antigens: application to diagnosis of tuberculosis infection and assessment of response to mycobacterial vaccines. Am J Med Sci 1997; 313: $364-371$.

13. Brock I, Munk ME, Kok-Jensen A, Andersen P. Performance of whole blood IFN-gamma test for tuberculosis diagnosis based on PPD or the specific antigens ESAT-6 and CFP-10. Int J Tuberc Lung Dis 2001; 5: 462-467.

14. Dye C, Scheele S, Dolin P, Pathania V, Raviglione MC. Consensus statement. Global burden of tuberculosis: estimated incidence, prevalence and mortality by country. WHO Global Surveillance and Monitoring Project. JAMA 1999 18; 282(7): 677-86.

15. Golden MP and Vikram HR. Extrapulmonary tuberculosis: An overview. Am Fam Physician 2005; 72(9): 1761-8.

16. Centers for disease control and prevention (CDC). Trends in tuberculosis- United States, 1998-2003. MMWR Morb Mortal Wkly Rep. 2004 Mar 19;53(10): 209-14. Erratum in MMWR Morb Mortal Wkly Rep. 2004 Mar 26; 53(11): 246.

17. Gledovic $\mathrm{Z}$, Vlajinac $\mathrm{H}$, Pekmezovic Chemotherapy and Infection. Int J Antimicrob Agents 2009; 34: 45-16
SK Baul, SMR Hossain, D Parvin et al

18. Pesut DP, Gledovic ZB, Grgurevic AD, Nagorni-Obradovic LJM and Adzic TN. Tuberculosis Incidence in Elderly in Serbia: Key Trends in Socioeconomic Transition. Croat Med J 2008; 49(6): 807-812.

19. Batut MJ. Communicable Diseases. In: Health Statistical Yearbook of Republic of Serbia 2012. Istitute of Public Health of Serbia [ISSN 2217-3714 online] 403-405. Available from: http: / / www.batut.org.rs/download/ health_indicators_rs.html.

20. Pelemis M, Stevanovic G, Pavlovic M, Nikolic S, Lavadinovic L, Milosevic B, et al. Importance of extrapulmonary tuberculosis during evaluation of patients with fever of unknown origin. Oral presentation; 26 ${ }^{\text {th }}$ International congress. 2011. Institute for infectious and tropical diseases, Clinical center of Serbia, Belgrade, Serbia.

21. Gupta BK, Bharat V, Bandyopadhyay D. Sensitivity, specificity, negative and positive predictive values of adenosine deaminase in patients of tubercular and non-tubercular serosal effusion in India. J Clin Med Res 2010; 2(3): 121-6.

22. SS Negi, R Anand, SF Basir. Protein antigen b (Pab) based PCR test in diagnosis of pulmonary and extra-pulmonary tuberculosis. Ind J Med Res 2006; 124: 81-8.

23. RC Kesarwani, A Pandey, A Misra, Polymerase chain reaction (PCR): Its comparison with conventional techniques for diagnosis of extrapulmonary tubercular disease. Indian J Surgery 2004; 66: 84-8.

24. P R Sharma, 3, S Jain, R N K Bamezai and P K Tiwari, Utility of Serum. LDH Isoforms in The Assessment of Mycobacterium Tuberculosis Induced Pathology in TB Patients of Sahariya Tribe. Ind J Clin Biochem, 2010; 25(1): 57-63.

25. Oznur Ak, Gul Dabak, Serdar Ozer, Attila saygi and Resat dabak. The Evaluation of the Quantiferon-TB gold Test in Pulmonary and Extrapulmonary tuberculosis. J. Infect Dis. 62, 149-151, 2009. 